\title{
Pain research and treatment in the gerontological context: A discussion of the issues
}

\author{
Margaret C Gibson $\mathrm{PhD}^{1}$, Michael R Thomas $\mathrm{PhD}^{2}$, Andrew J Cook $\mathrm{PhD}^{3}$
}

MC Gibson, MR Thomas, AJ Cook.

Pain research and treatment in the gerontological context: A discussion of the issues.

Pain Res Manage 1998;3(2):91-96.

An appreciation of the context in which gerontological health care occurs is crucial to the successful and ethical collection, interpretation and utilization of data on the pain experiences of elderly people. Important contextual considerations for researchers and clinicians who are addressing pain in the elderly are identified. It is argued that topics that are the focus of current attention in the field of pain and ageing, such as the effect of dementia on memory for pain and the responsiveness of the elderly to psychosocial pain treatment, would be clarified by an understanding of the broader sociopolitical context in which ageing occurs.

Key Words: Elderly, Memory, Pain, Treatment

\section{Recherche sur la douleur et son traitement dans le contexte gérontologique : les questions à débattre}

RÉSUMÉ : Une évaluation du contexte dans lequel les soins de santé gérontologiques sont dispensés est indispensable pour collecter, interpréter et utiliser des données valables et respectant les critères d'éthique, sur l'expérience de la douleur chez les personnes âgées. Des questions contextuelles importantes pour les chercheurs et les cliniciens qui s'intéressent à la douleur chez la personne âgée sont identifiées. Les auteurs soutiennent que les sujets sur lesquels l'on se concentre actuellement dans le domaine de la douleur et du vieillissement, comme l'effet de la démence sur la mémoire pour la douleur et la réponse de la personne âgée au traitement psychosocial de la douleur, seraient éclaircis par une meilleure compréhension du contexte sociopolitique dans lequel la vieillesse survient.
$\mathrm{Pa}_{\mathrm{r} \text { mand }}$ ain in the elderly is attracting increasing attention from researchers. As a complement to this attention, it is important that we concurrently focus on relevant aspects of the prevailing sociopolitical culture because neither empirical data nor clinical outcomes can be understood separate from the context in which they are generated (1). In particular, an appreciation of the ageing process and of gerontological health care is crucial to the successful and ethical collection, interpretation and utilization of data on the pain experiences of elderly people.
Important contextual issues to be addressed when designing, implementing and disseminating research with elderly participants include obtaining informed consent for research participation where cognition is impaired, the possibility that sensitive clinical issues may be uncovered during the course of a research investigation, the identification of appropriate outcome measures, the importance of life stage and cohort factors, the responsiveness of the health care system to the needs of the elderly and the effect of ageism. We present an overview of these issues, recognizing that other equally im-

\footnotetext{
${ }^{1}$ Veterans Care Program, Parkwood Hospital, London, Ontario; ${ }^{2}$ Department of Psychology, University of Manitoba, Winnipeg, Manitoba;

${ }^{3}$ Vancouver Pain Clinic, Vancouver, British Columbia

Correspondence: Dr M Gibson, Veterans Care Program, Parkwood Hospital, 801 Commissioners Road East, London, Ontario N6C 5J1.

Telephone 519-685-4292 ext 2708,fax 519-685-4031, e-mail m.gibson@parkwood.Iondon.on.ca

Received for publication October 30, 1997. Accepted March 31, 1998
} 
portant issues no doubt could have been selected for discussion. To illustrate the interplay between research findings on pain and ageing and the context in which elderly adults receive their health care, we refer to two topics that are the focus of current attention in the field of pain and ageing: the effect of dementia on memory for pain and the responsiveness of the elderly to psychosocial pain treatment.

\section{PAIN RESEARCH IN THE GERONTOLOGICAL CONTEXT}

\section{Informed consent}

In most jurisdictions there are legal criteria and standards for establishing informed consent to treatment irrespective of the patient's cognitive status. However, protocols for obtaining informed consent for research participation are less formalized. Ensuring one has informed consent for research can be a complex task even with participants who are cognitively aware. The complexities are compounded where members of the potential study sample are vulnerable, secondary to conditions such as impaired cognitive abilities (2). Elderly individuals who suffer from dementing disorders are at significant risk of marginalization in the research domain $(3,4)$. On the one hand, without vigilance on the part of researchers, family and advocates, they may become unwilling (or unwitting) research participants (5). That is, consent may be granted on their behalf, without due consideration given to the choices they would have made were they able (judging from their past behaviours). Alternatively, consent may be granted by a substitute decision-maker, while assent is ignored. Violations are particularly likely where fluctuating cognitive abilities create difficulties for research schedules and routines that involve repeated measurement and/or ongoing intervention. Clinicians and family members speak of individuals with dementia as having 'good days' and 'bad days'. In the research context, this can translate into days on which an individual willingly cooperates with a research protocol and days on which he or she most emphatically does not. Ethical standards clearly exhort the researcher to develop processes for confirming continuing agreement for research participation in such situations (6).

As noted in the recent report from the National Forum on Health, "Research should benefit all people, and populations should not be treated with interventions that have not been tested on them. Legal restrictions designed to protect population groups from research risk may actually expose them to greater risk" (7). Elderly adults with impaired cognitive abilities are at risk of unjust exclusion from participation in research that is directly relevant to their interests, due to the difficulties inherent in orchestrating their participation (8). Sachs and colleagues (9) highlighted the difficulties in conducting research in nursing home settings, noting that such research typically requires more time, additional discussions, adaptations to standard consent forms and procedures, and modification of data collection instruments. Innovative information strategies that have been used with this population include use of audiotapes, videotapes and 'story book' formats to explain a study or a procedure. Potential differences of opinion between researchers and clinicians in these settings about the role of research need to be addressed openly, to ensure that residents are neither exploited nor unjustly excluded from the opportunity for research participation.

\section{Sensitive issues}

A related concern pertains to the researcher's ethical responsibilities when a research investigation yields unexpected information that may have legal, professional or public interest implications, such as elder abuse. Elder abuse increasingly is being recognized as a significant societal concern. The prevalence rate for elder abuse and neglect among Canadian seniors is estimated at 4\% (10). A recent monograph sponsored by the Canadian Association on Gerontology (11) offers recommendations for clinicians, researchers and educators to enhance their detection of, and responsiveness to, this issue. The authors acknowledge that legislative support for reporting and intervention practices is both sparse and regionally fragmented. Nonetheless, research teams are advised to be prepared for the possibility that elder abuse may be suspected or detected. A contingency plan that is responsive to both professional ethics and local expectations (eg, institutional abuse policies) should be in place (12).

In a similar scenario, undiagnosed depressive disorders or significant untreated pain concerns may be detected when interviewing elderly people who are participating in a research study. Regarding the communication of clinically relevant information, investigators should consider how, to whom and under what circumstances it will be disseminated.

\section{Measuring outcomes}

Different outcomes and different risk factors may be relevant for different populations (13). The criteria for evaluating pain treatment success with older adults may differ from those used with younger adults. For example, vocational issues often are not as important as involvement in social and community activities. Gerontological health care typically focuses on improvement in function and amelioration of symptoms, rather than on cure or symptom resolution $(14,15)$. When productive activity is used as an outcome criteria in treatment evaluations with the elderly, it should be broadly defined to include paid employment, housework, volunteer work, yard work and training/education (16). Other measures used with younger adults are usually applicable to the elderly (eg, reduced incidence and intensity of pain, increased activity and improved subjective well-being) (16). Generally, however, treatment should target individually established goals that are specified during the assessment process and have meaning to the elderly person. With elderly patients, clinicians are urged to be especially attentive to the individual's belief in his or her ability to complete tasks. Goals for various components of a pain management program (eg, reducing reliance on medications and decreasing tension and anxiety) may seem straightforward to the therapist but completely unrealistic to the elderly pain sufferer (17). Research on treatment effectiveness needs to reflect these sensitivities in the outcomes that are selected for evaluation, to avoid misinter- 
pretations about the responsiveness of the elderly to pain treatment.

The involvement of family members and other caregivers in treatment is crucial with elderly patients (18). Where indicated, sessions should be held with members of the older person's support network to ensure that all involved understand the components of treatment and can work together to maximize follow-through. If adequate support is not available, it may be appropriate to arrange, with the elderly person's endorsement, home care visits, involvement in a community centre, support group participation, etc. Researchers may likewise find their efforts more acceptable and effective when they appropriately (ie, with permission) access the elderly person's support system to assist with follow-through on research participation.

\section{Life stage and cohort factors}

The literature on pain treatment for the elderly reveals strong support for adapting psychosocial pain treatments to reflect the historical and situational circumstances of the elderly. For example, it is recognized that elderly individuals with chronic pain usually have developed a repertoire of personally effective coping strategies, and these should be incorporated into planned interventions (19). It has been argued that it is especially important when working with older adults to be sensitive to potential cohort or generational differences in beliefs regarding pain, coping and acceptable treatments (20, but see 21 for an alternate view). Clinicians are urged to respect the role of 'tried and true' home remedies that may have provided an important source of comfort to the elderly pain sufferer, and to be willing to incorporate these into a self-management program for chronic pain (20).

The unique social context of older adults is recognized. This includes formal (eg, health care system and social service agencies), informal (eg, family and friends) and semiformal (eg, Church, club, family doctor, pharmacist) networks (18). Social support can provide considerable buffering power against stressors (22) and plays a critical role in meeting the health needs of the elderly (23). Older people tend to supplement medical opinion with lay consultation within their social support network (24). This lay consultation may reflect cohort differences in attitudes towards health care and the medical system.

Although pain sufferers of all ages encounter stressors that negatively influence their pain perceptions and functioning, the stressors faced by the elderly are known to differ from those faced by younger adults (25). Common stressors for the elderly include retirement, reduced income, difficulty adjusting to rapid technological changes in society, more frequent illness and chronic health conditions, and shrinkage of social network through loss of spouses, siblings and friends. Often, pain management can be facilitated by addressing these issues. A thorough psychosocial history is an essential part of the assessment process with individuals of all ages.

What the broader gerontological literature can be taken to indicate is that at this stage of our understanding, there is a need for qualitative and well as quantitative research on how the elderly are affected by their pain experiences within their unique life circumstances, to complement the current attention to age differences in pain sensitivities and behaviours.

\section{Systemic factors}

Given the prevalence of pain-related medical conditions in the elderly population (26), one might expect to see substantial participation by the elderly in pain management clinics. This outcome has not been realized, however. Rather, pain management clinics appear to be notably underutilized by older adults (27). A contributing factor may be that these services are, in reality, not always readily available. A survey of Canadian pain clinics conducted in the late 1980s (28) yielded 37 sites, and it is unlikely that the numbers have increased in the current health care climate of cutbacks and downsizing. Older Canadians may have more difficulty than their younger counterparts in accessing a relatively scarce resource due to secondary factors such as transportation difficulties and mobility limitations. The extent to which pain clinic attendance is disproportionate, relative to demographic patterns, warrants further clarification (29). One response to this situation is to develop pain clinics that cater exclusively to the needs of the elderly. Initial outcome data on specialty pain clinics for elderly patients are promising (30).

The majority of the elderly, however, are likely to receive treatment for their pain problems, along with their other ailments, in primary care $(31,32)$. Unfortunately, the medical model alone is inadequate as a response to the multiple interacting medical, psychological and social factors that contribute to functional difficulties in a gerontological population. As a result, the elderly may not receive adequate treatment for their pain conditions in these settings (33). The factors that create, perpetuate and could remedy this situation warrant more focused investigation.

\section{Ageism}

The extent to which the health care system meets the pain control needs of the elderly - in terms of both service availability and targeted outcomes - is a reflection of broader societal values. The Encyclopedia of Aging, Second Edition (34) defines ageism as "a process of systematic stereotyping and discrimination against people because they are old, just as racism and sexism accomplish this for skin color and gender". Ageism creates a bias in favour of youth as the prime target for health intervention and provides a justification for the exclusion of the old (35). Beliefs about the etiology of pain and its relationship to the ageing process, on the part of both clients and health care professionals, are important (20). Cook and Thomas (19) found that 38\% of elderly individuals reporting chronic pain stated that their physicians had attributed their pain to ageing. Discussion of these types of beliefs with clients is a valuable component of intervention because research has established that client-caregiver agreement contributes to better treatment outcomes, greater compliance with prescribed regimens and greater patient satisfaction (20). Researchers, no less than clinicians, need to guard against the insidious effects of ageism. 


\section{A CONTEXTUAL CONSIDERATION OF CURRENT RESEARCH QUESTIONS}

Research on pain in the elderly and treatment for the pain concerns of the elderly occur within the larger sociopolitical context of gerontological health care and ageing in society. We consider the interpretation and utilization of two current lines of study: pain memory and dementia, and the responsiveness of elderly pain sufferers to psychosocial pain treatment. Our intent is not to imply that consideration of context is in any way unique to these lines of research, but rather to raise awareness of the issues.

\section{Pain memory in the elderly}

The gerontological literature supports two observations about ageing that are critical to the field of pain research and management. First, many older adults experience the damaging affects of a progressive dementia (36). Dementia is characterized by multiple cognitive deficits, most notably memory impairment (37). The incidence of moderate to severe dementia is approximately 5\% to $7 \%$ between 65 and 80 years of age, and $20 \%$ for those over 80 years old (38). The ongoing demographic shift towards the older segment of the population is expected to result in a significant increase in the absolute numbers of elderly people with dementia over the next decade (36). It has been reported that over half of the elderly who reside in institutional care facilities have some degree of dementia (38). Also, for many older adults, increased age is associated with chronic conditions that are accompanied by pain (26). Roy and Thomas (39) reported that $83 \%$ of elderly people in institutions report pain. Similarly, Ferrell et al (40) reported a $71 \%$ prevalence rate of pain in nursing home residents.

If over half of the elderly in institutional care have pain conditions, and nearly half have some degree of dementia, the likelihood of high comorbidity is an obvious potential problem for the reliability of pain report in this population. Research on dementia and pain memory is sparse; however, one report suggests that while reliability may be compromised, validity is intact. Parmelee and colleagues (41) solicited pain complaints in a study involving 758 long term care residents. Spontaneous pain reporting was diminished in those with cognitive impairments, relative to their cognitively intact peers. Importantly, pain recall accuracy, as measured by physician expectations based on disease status, did not differ as a function of cognitive status.

In their review of the pain and dementia literature, Ferrell et al (42) noted that several factors, including disease-related problems in language production, memory impairments and/or neurophysiologically based influences on sensitivity to pain, may contribute to unreliable pain recall. They concluded, "That dementia impacts on the experience and report of pain seems very probable.... What is not clear is the extent and direction of this influence." In a related vein, Anand and Craig (43) addressed the need to revisit the accepted and standardized definition of pain (ie, "pain is defined as an unpleasant sensory or emotional experience associated with actual or potential tissue damage or described in terms of such damage"
[44]), to ensure that we are not inadvertently marginalizing individuals for whom the availability of reliable, verbal selfreport is uncertain.

Empirical and conceptual issues such as these have an obvious interface with the sociopolitical world of health care delivery. The elderly are undertreated for pain in general, and the presence of dementia exacerbates this unfortunate circumstance (45). There is an elevated risk of undertreatment when sufferers are ineffective communicators (46), as are many of the elderly with dementia. The perception that memory for pain is impaired in individuals with dementia can exacerbate the situation, with serious implications for quality of life (47). Efforts to alleviate pain and suffering often do not receive the priority they deserve from caregivers when it is thought that the care recipient is 'spared' by virtue of his or her lack of memory ability.

Successful efforts to translate research on pain and dementia into gerontological health care practice hinge on uniting knowledge from both domains. The goal is for research results to affect clinical outcomes positively, rather than to entrench negative stereotypes further. An appreciation of the complex biopsychosocial nature of pain experience is essential so that research findings on the factors that may mediate different aspects of pain perception (eg, cognitive status) are understood. Gerontological knowledge, in turn, promotes awareness that those with dementia are members of a group at high risk of inadequate pain control (ie, the elderly) and that the most salient feature of the dementias is their heterogeneity of presentation (48). The pattern of spared and impaired functions varies widely among afflicted individuals and across time within individuals as the disease progresses. Generalizations are ineffective at the level of planning care for an individual. The importance of multidimensional, flexible and sensitive pain assessment practices that accommodate the fluctuating abilities of the person with dementia cannot be overemphasized (49).

\section{Responsiveness to psychosocial pain treatment}

There is a growing body of literature demonstrating that older adults benefit from multidisciplinary pain treatments. For example, the elderly have been shown to benefit from psychosocial and behavioural treatments such as cognitivebehavioural pain programs (50), biofeedback (51) and relaxation training (52). The general consensus from the literature appears to be that older and younger adults are similarly able to benefit from psychosocial pain treatments $(53,54)$.

The idea that age, in and of itself, might affect responsiveness to pain treatment has an ageist flavour. The elderly comprise the most heterogeneous segment of the population in terms of health, function, abilities and interests. Moreover, depending on the cut-off criteria, those classified among the ranks of the elderly may differ in chronological age from one another by as much as 30 or 40 years (55). Ageism is reflected in the common assumption that older people are more difficult than younger people to treat in some way, such as requiring more therapist time or requiring highly specialized treatment programs. Kee and colleagues (16) evaluated this 
assumption as part of an outcome study of their multidisciplinary chronic pain rehabilitation program. Clinician ratings on compliance, amount of time and attention, need for modification of treatment protocols and the extent of improvement, indicated that community dwelling older adults responded well and were not harder to treat than younger adults. No treatment components were identified as providing special problems for them.

The contextual question is what factors (such as the presence of dementia or other disease states, social support factors, expectations or systemic barriers) affect responsiveness to psychosocial pain treatments, and in what ways? From this perspective, chronological age is treated as a covariate, rather than as a potential explanatory variable (55). Multidisciplinary clinical efforts to improve pain treatment in the elderly rely on this contextualized approach, with more attention paid to issues such as comorbidity, functionality, polypharmacy, mediating variables (eg, social support) and the significance of cohort and life stage factors, than to age per se (17).

In this approach, contributions of age and cognitive status are clearly differentiated. An individual's particular pattern of cognitive deficits is interpreted within a holistic understanding of strengths and limitations. This helps to control for the risk that elderly individuals with mild cognitive impairment will be excluded from potentially beneficial treatments (49). The greater incidence and severity of cognitive impairment among the elderly is recognized as an additional consideration (but not an exclusionary criterion) for treatment planning. Cook (56) evaluated a cognitive-behavioural pain management program for elderly nursing home residents against an attention/support control treatment. Results revealed that the residents who received the cognitivebehavioural training reported less pain and pain-related disability, and that these effects were maintained at four months' follow-up. Residents participating in the program ranged from 61 to 98 years old and included individuals with mild cognitive impairment. The willingness of an elderly individual to report pain and his or her cooperation with the assessment and intervention were recognized as more important than cognitive status alone. Effective and acceptable methods for communicating about pain can generally be developed once adequate rapport is established.

\section{REFERENCES}

1. Keating NC, Fast JE. Bridging policy and research in Eldercare. Can J Aging 1997;16(Suppl):22-41.

2. Weisstub DN, Arboleda-Florez J, Tomossy GF. Establishing the boundaries of ethically permissible research with special populations. Health Law Canada 1996;17:45-63.

3. Beland F. Building Canadian gerontology: A springboard for international recognition? Can J Aging 1997;16:6-10. (Edit)

4. Kerserlingk EW, Glass K, Kogan S, Gauthier S. Proposed guidelines for the participation of persons with dementia as research subjects. Perspect Biol Med 1995;38:319-62.

5. Wicclair MR. Ethics and the Elderly. New York: Oxford University Press, 1993.

6. National Council of Bioethics in Human Research. Facilitating ethical research: Promoting informed choice - Discussion document. NCBHR Communique 1996;7(Suppl):1-28.
From a contextual perspective, the question whether the elderly can benefit, or benefit as much as their younger aged peers, from psychosocial pain management strategies is answered, in part, by how sensitive and creative we are in tailoring interventions to the needs of the individual client. Richardson (57) provides suggestions for enhancing compliance with medical regimens for elderly patients, and these can apply equally to all types of pain management interventions. These suggestions include pacing the presentation, task relevance and difficulty level to the elderly person's ability; increasing time for the person to study visual material and to respond; slowing speech pace; providing additional organizational assistance to facilitate memory; nurturing the person's hope in their care and treatment; and ensuring that support is available. Asking people to repeat the rationale and instructions for treatment is an effective way of addressing concerns regarding attention, hearing or comprehension. Not all interventions have application with all clients, but different interventions have application with individuals of differing abilities, regardless of age. The astute practitioner is familiar with a broad range of compensatory strategies to facilitate treatment implementation.

The life stage and cohort issues discussed earlier herein can be incorporated into treatment planning to maximize the fit between psychosocial pain treatments and the needs of the elderly client. The challenge is to incorporate these approaches at the access points in the health care system where they can do the most good. In the case of the elderly at this time, this is more likely to be at the primary care and residential care levels than in specialty pain clinics.

\section{SUMMARY}

Pain in the elderly is a significant clinical concern. Research indicates that the pain problems of elderly people are responsive to treatment. Cognitive impairment is not an insurmountable barrier to assessment and intervention. Research activities and clinical service appear to be most readily integrated and most useful for furthering health and well-being when combined with an appreciation of relevant contextual issues. The processes of ageing and the dynamics of gerontological health care are the contextual bedrock on which a greater understanding of pain in the elderly will be built.

7. National Forum on Health. Creating a culture of evidence-based decision making in health. In: National Forum on Health: Synthesis Reports and Issues Papers. Ottawa: Ministry of Public Works and Government Services, 1997:14.

8. Larsen E. Exclusion of certain groups from clinical research. J Nurs Scholar 1994;26:185-90.

9. Sachs GA, Rhymes J, Cassel CK. Biomedical and behavioural research in nursing homes: Guidelines for ethical investigations. J Am Geriatr Soc 1993;41:771-7.

10. Podnieks E, Pillemer K, Nicholson JP, Shillington T, Frizzel AF. National Survey on Abuse of the Elderly in Canada. Toronto: Ryerson Polytechnical Institute, 1990.

11. MacLean M, ed. Abuse and Neglect of Older Canadians: Strategies for Change. Toronto: Thompson Educational Publishing, 1995.

12. National Council of Bioethics in Human Research. Ethics of Clinical 
Trials for Research Ethics Boards: Proceedings of a National Workshop. NCBHR Communique 1991;2:1-23.

13. Black JS, Kapoor W. Health promotion and disease prevention in older people: Our current state of ignorance. J Am Geriatr Soc 1990;38:168-72.

14. Satin DG. Emotional and cognitive issues in the care of the aged. In: Satin DG, ed. Clinical Care of the Aged Person. New York: Oxford University Press, 1994:62-107.

15. Wallace RB, Rohrer JE. Aging, quantitative health status assessment and the effectiveness of medical care. In: Kane RL, Evans JG, MacFadyen D, eds. Improving the Health of Older People: A World View. Oxford: Oxford University Press, 1990:91-106.

16. Kee WG, Middaugh SJ, Pawlick KL. Persistent pain in the older patient: Evaluation and treatment. In: Gatchel R, Turk D, eds. Psychological Approaches to Pain Management. New York: Guilford, 1996:371-402.

17. Gibson SJ, Farrell MJ, Katz B, Helme RD. Multidisciplinary management of chronic nonmalignant pain in older adults. In: Ferrell BR, Ferrell BA, eds. Pain in the Elderly. Seattle: IASP Press, 1996:91-100.

18. Roy R, Thomas M, Cook AJ. Social context of elderly chronic pain patients. In: Ferrell BR, Ferrell BA, eds. Pain in the Elderly. Seattle: IASP Press, 1996:111-8.

19. Cook AJ, Thomas MR. Pain and the use of health services among the elderly. J Aging Health 1994;6:155-72.

20. Cook AJ, Roy R. Attitudes, beliefs, and illness behaviour. In: Roy R, ed. Chronic Pain in Old Age: An Integrated Biopsychosocial Perspective. Toronto: University of Toronto Press, 1995:20-37.

21. Gagliese L, Melzack R. Lack of evidence for age differences in pain beliefs. Pain Res Manage 1997;2:19-27.

22. MacRae H. Strong and enduring ties: Older women and their friends. Can J Aging 1996;15:374-92.

23. Denton M. The linkages between informal and formal care of the elderly. Can J Aging 1997; 16:30-50.

24. Edwardson SR, Dean KJ, Brauer DJ. Symptom consultation in lay networks in an elderly population. J Aging Health 1995;7:402-16.

25. Ensel WM, Peek MK, Lin N, Lai G. Stress in the life course: A life history approach. J Aging Health 1996;8:389-416.

26. Ross MM, Crook JM. Pain in later life: Present state of knowledge. In: Roy R, ed. Chronic Pain in Old Age: An Integrated Biopsychosocial Perspective. Toronto: University of Toronto Press, 1995:3-19.

27. Roy R. A psychosocial perspective on chronic pain and depression in the elderly. Soc Work Health Care 1987;12:27-36.

28. Catchlove RFH, Hoirch AM. Survey of Canadian pain centres: A preliminary report. Pain Clin 1989;2:231-7.

29. Gagliese L, Melzack R. Chronic pain in elderly people. Pain 1997;70:3-14.

30. Sandin KJ. Specialized pain treatment for geriatric patients. Clin J Pain 1993;9:60.

31. Barer ML, Evans RG, Hertzman C. Avalanche or glacier: Health care and demographic rhetoric. Can J Aging 1995;14:193-224.

32. Black C, Roos NP, Havens B, MacWilliams L. Rising use of physician services by the elderly: The contribution of morbidity. Can J Aging 1995; 14:225-44.

33. Crook J, Weir R, Tunks E. An epidemiological follow-up survey of persistent pain sufferers in a group family practice and specialty pain clinic. Pain 1989;36:49-61.

34. Maddox GL, ed. The Encyclopedia of Aging, 2nd edn. New York: Springer, 1995:35-6.
35. Hirdes JP, Carpenter GI. Health outcomes among the frail elderly in communities and institutions: Use of The Minimum Data Set (MDS) to create effective linkages between research and policy. Can J Aging 1997;16(Suppl):53-69.

36. Youngjohn JR, Crook TH III. Dementia. In: Carstensen LL, Edelstein BA, Dornbrand L, eds. The Practical Handbook of Clinical Gerontology. Thousand Oaks: Sage, 1996:239-54.

37. American Psychiatric Association. Diagnostic and Statistical Manual of Mental Disorders, 4th edn. Washington: American Psychiatric Association, 1994.

38. Herrmann, N. Dementia. In: Conn DK, Herrmann N, Kaye A, Rewilak D, Robinson A, Schogt B, eds. Practical Psychiatry in the Nursing Home. Seattle: Hogrefe and Huber, 1992:43-62.

39. Roy R, Thomas M. Survey of chronic pain in an elderly population. Can Fam Physician Med Fam Can 1986;32:513-6.

40. Ferrell BA, Ferrell BR, Osterweil D. Pain in the nursing home. J Am Geriatr Soc 1990;38:409-14.

41. Parmelee PA, Smith B, Katz IR. Pain complaints and cognitive status among elderly institution residents. J Am Geriatr Soc 1993;41:517-22.

42. Ferrell MJ, Katz B, Helme RD. The impact of dementia on the pain experience. Pain 1996;67:7-15.

43. Anand KJS, Craig KD. New perspectives on the definition of pain. Pain 1996;67:3-6. (Edit)

44. International Association for the Study of Pain Task Force on Taxonomy. Classification of Chronic Pain, 2nd edn. Seattle: IASP Press, 1994:210.

45. Fulmer TT, Mion LC, Bottrell MM, Faculty N. Pain management protocol. Geriatr Nurs 1996;17:222-7.

46. Simons W, Malabar R. Assessing pain in elderly patients who cannot respond verbally. J Adv Nurs 1995;22:663-9.

47. Gibson MC. Improving pain control for the elderly patient with dementia. Am J Alzheimer's Dis 1998;13:10-4.

48. Nebes RD. Cognitive dysfunction in Alzheimer's disease. In: Craik FIM, Salthouse TA, eds. The Handbook of Aging and Cognition. Hillsdale: Lawrence Erlbaum Assoc, 1992:373-446.

49. Herr KA, Mobily PR. Pain management for the elderly in alternate care settings. In: Ferrell BR, Ferrell BA, eds. Pain in the Elderly. Seattle: IASP Press, 1996:101-9.

50. Puder RS. Age analysis of cognitive-behavioural group therapy for chronic pain outpatients. Psychol Aging 1988;3:204-7.

51. Middaugh SJ, Woods E, Kee WG, Harden RN, Peters JR. Biofeedback-assisted relaxation training for the aging chronic pain patient. Biofeed Self-Regulation 1991;16:361-77.

52. Arena JG, Hightower NE, Chang GC. Relaxation therapy for tension headache in the elderly: A prospective study. Psychol Aging 1988;3:96-8.

53. Cutler RB, Fishbain DA, Rosomoff RS, Rosomoff HL. Outcomes in treatment of pain in geriatric and younger age groups. Arch Phys Med Rehab 1994;75:457-64.

54. Sorkin BA, Rudy TE, Hanlon RB, Turk DC, Steig RL. Chronic pain in old and young patients: Differences appear less important than similarities. J Gerontol 1990;45:64-8.

55. Gibson, MC. Pain in the elderly: Psychosocial issues. Curr Rev Pain 1998;2:29-40.

56. Cook AJ. Cognitive-behavioural pain management for elderly nursing home residents. J Gerontol Psychol Sci 1998;53B:51-9.

57. Richardson JL. Perspectives on compliance with drug regimens among the elderly. J Compliance Health Care 1986;1:33-46. 


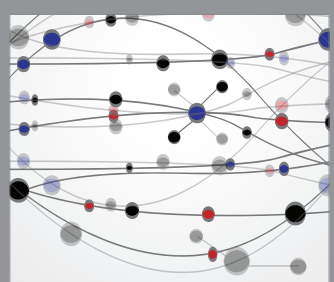

The Scientific World Journal
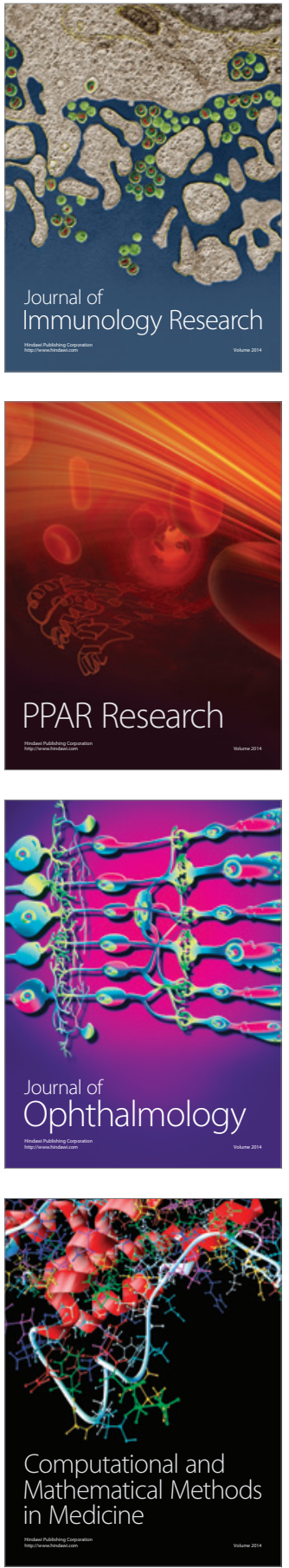

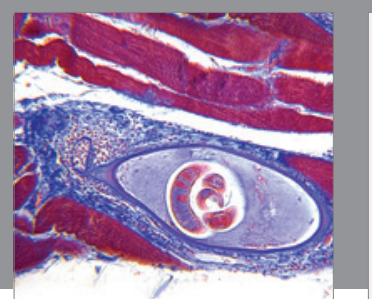

Gastroenterology Research and Practice

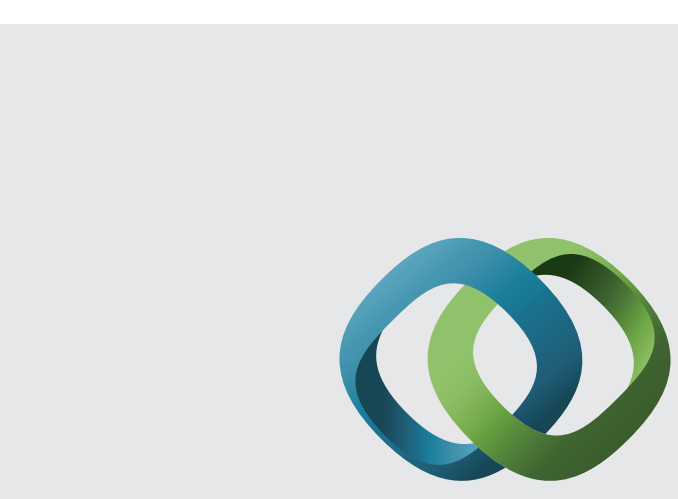

\section{Hindawi}

Submit your manuscripts at

http://www.hindawi.com
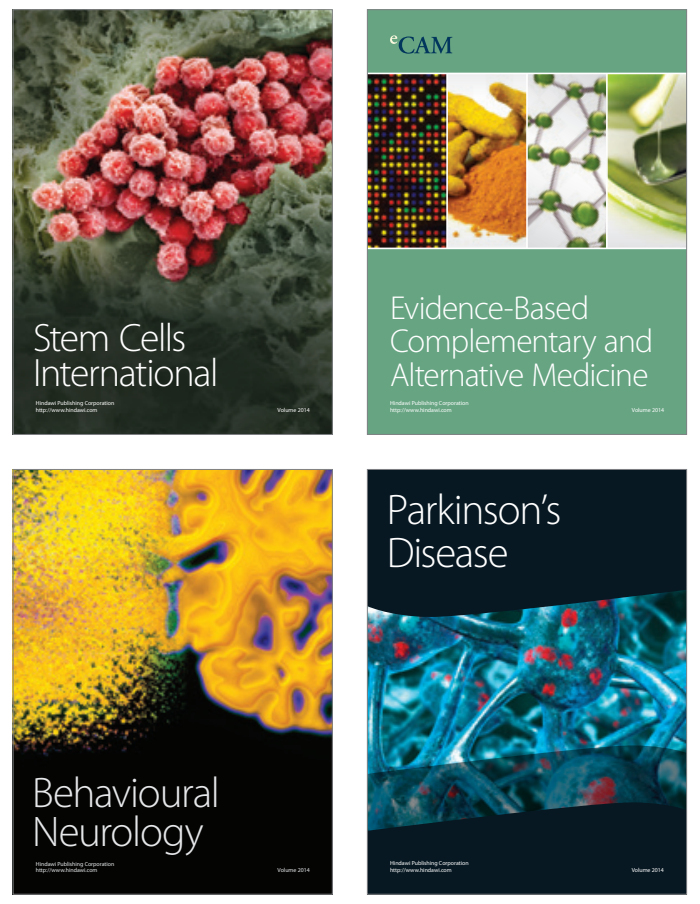
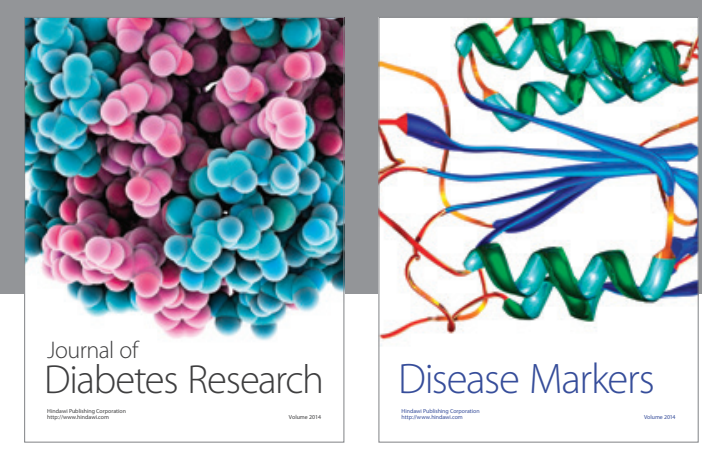

Disease Markers
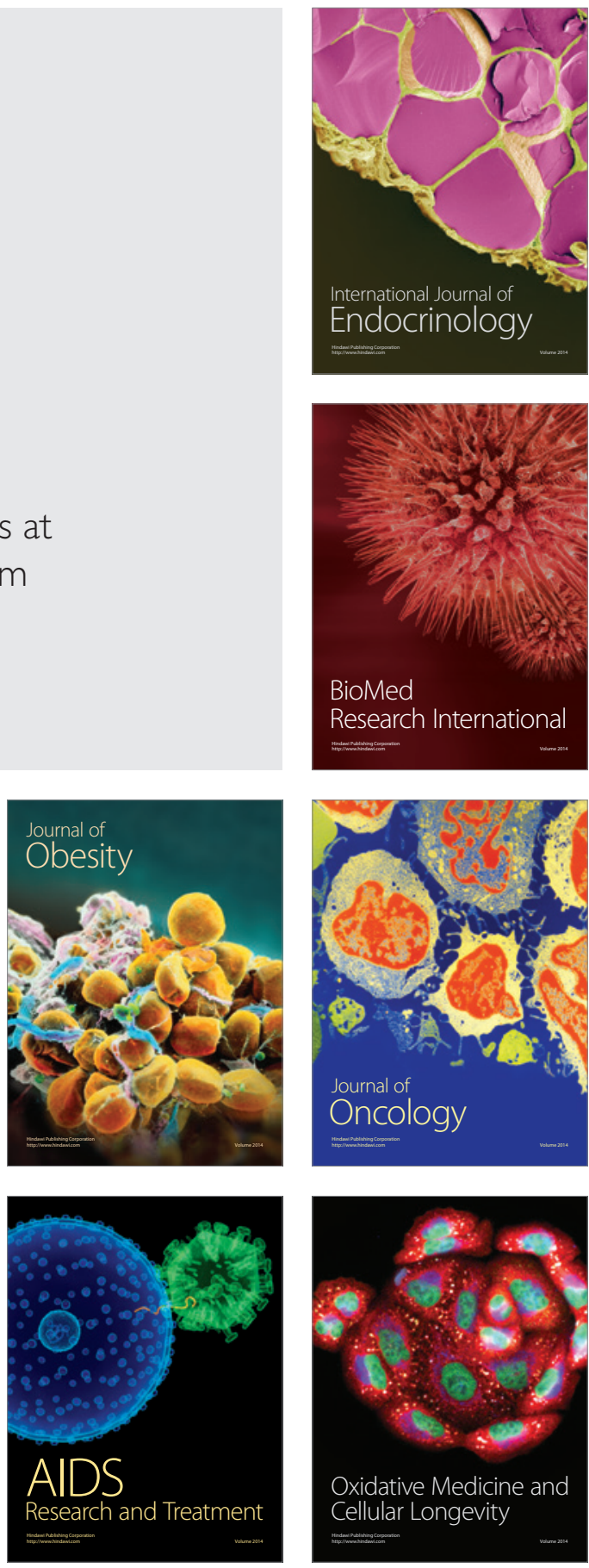